\title{
Assessment of the impact and reorganization of interventional pulmonology services at a tertiary care centre during nationwide lockdown for COVID-19 pandemic
}

\author{
Rahul Tyagi", Saurabh Mittal", Karan Madan, Anant Mohan, Vijay Hadda, Pawan Tiwari, Randeep Guleria \\ Department of Pulmonary, Critical Care and Sleep Medicine, All India Institute of Medical Sciences (AIIMS), New Delhi, \\ India
}

${ }^{*} R T$ and SM contributed equally and are the joint first authors of this manuscript

\begin{abstract}
COVID 19 pandemic has brought about a sea change in health care practices across the globe. All specialities have changed their way of working during the pandemic. In this study, we evaluated the impact of COVID-19 on the practice of interventional pulmonology at our centre. All interventional pulmonology proce-
\end{abstract}

Correspondence: Dr. Saurabh Mittal, Assistant Professor, Department of Pulmonary, Critical Care and Sleep Medicine, All India Institute of Medical Sciences (AIIMS), Ansari Nagar, New Delhi 110029, India. Tel. +91.11.26546346.

E-mail: saurabh_kgmu@yahoo.co.in

Authors' contributions: RT, initial manuscript preparation; SM, conceived the idea and final manuscript preparation; KM, data analysis; AM, manuscript revision; VH, PT, RG, manuscript revision. All the authors have read and approved the final version of the manuscript and agreed to be accountable for all aspects of the work.

Ethics approval and consent to participate: Ethical clearance was obtained from the Institute Ethics Committee and written informed consent for the procedure was obtained.

Consent for publication: This is a retrospective analysis, so consent for publication was not obtained from patients.

Availability of data and material: The datasets used and/or analyzed during the current study are available from the corresponding author on reasonable request.

Conflict of interest: The authors declare that they have no competing interests, and all authors confirm accuracy.

Key words: Bronchoscopy; COVID-19; SARS CoV-2; EBUS-TBNA.

Received for publication: 29 September 2020.

Accepted for publication: 26 November 2020.

${ }^{\circ}$ Copyright: the Author(s), 2021

Licensee PAGEPress, Italy

Monaldi Archives for Chest Disease 2021; 91:1615

doi: 10.4081/monaldi.2021.1615

This article is distributed under the terms of the Creative Commons Attribution Noncommercial License (by-nc 4.0) which permits any noncommercial use, distribution, and reproduction in any medium, provided the original author(s) and source are credited. dures done during the four months after implementation of lockdown were evaluated retrospectively for patient demographics, clinical diagnosis, indication for procedure and diagnostic accuracy. The changes in practices, additional human resources requirement, the additional cost per procedure and impact on resident training were also assessed. Procedures done during the month of January 2020 were used as controls for comparison. Twenty-two flexible bronchoscopies (75.8\%), four semirigid thoracoscopies (13.7\%) and three EBUS-TBNAs (10.3\%) were carried out during four-month lockdown period as compared to 174 during January 2020. Twenty-three of the procedures were for the diagnostic indication $(79 \%)$, and six were therapeutic $(20.6 \%)$. The diagnostic yield in suspected neoplasm was $100 \%$ while for suspected infections was $58.3 \%$. The percentage of independent procedures being done by residents reduced from $45.4 \%$ to $0 \%$. The workforce required per procedure increased from 0.75 to $4-8$, and the additional cost per procedure came out to be 135 USD. To conclude, COVID 19 has impacted the interventional pulmonology services in various ways and brought about a need to reorganize the services, while also thinking of innovative ideas to reduce cost without compromising patient safety.

\section{Introduction}

Coronavirus disease 2019 (COVID 19) outbreak has caused significant mortality and morbidity around the globe [1]. The infection spreads by direct transmission through infectious droplets from coughing or sneezing onto a mucous membrane, direct contact and fomites [2]. The pandemic has severely impacted the healthcare systems across the world, especially in developing countries like India. Till $12^{\text {th }}$ September 2020, more than 4.6 million cases and more than 75,000 deaths have been reported in India even though there were initial observations that tuberculosis endemic countries may have less burden [3-5]. The pandemic has changed the healthcare practices across the world. The disease has significantly affected health care workers (HCWs) also, with various studies documenting infection rate in HCWs, ranging from $3.8 \%$ to $29 \%$ [6,7]. Use of personal protective equipment such as gowns, surgical masks or respirators, face shields and gloves along with hand hygiene, are recommended for personal protection in different patient care areas [2].

Procedures such as bronchoscopy and thoracoscopy are considered aerosol-generating, and there are concerns regarding the high risk of virus transmission. Recently, American college of 
chest physicians (ACCP) has provided guidance regarding bronchoscopy in COVID-19 [8]. The pandemic has also affected the residency programs and is causing a lack in academic, surgical and procedural training.[9] Access of the resident doctors to departments has been limited. The procedures which are restricted are those, which were mostly performed by residents. Clinical case discussions and meetings are being cancelled to avoid gathering. These have definitely affected training, and similar disruptions are applicable to other departments also.

As we realize, COVID 19 has affected all aspects of health care ranging from changes in patient management protocols to enhanced precautions by HCWs and disruptions in medical training; we conducted this study to evaluate the impact of the nationwide lockdown (between 24 Mar 2020 to 23 Jul 2020) due to COVID 19 on interventional pulmonology services at a tertiary care centre in north India and how the system evolved to meet the ever-changing requirements.

\section{Methods}

\section{Study design and patients}

We conducted a retrospective study at a tertiary care teaching centre in Delhi, India. All patients who underwent bronchoscopy, endobronchial ultrasound-guided transbronchial needle aspiration (EBUS-TBNA) or thoracoscopy during the lockdown period between 24 Mar 2020 to 23 Jul 2020 were included in the study. Patient records were reviewed for demographic data, clinical diagnosis, indication for the procedure, testing for COVID-19 and whether a diagnosis was achieved.

\section{Preparation of bronchoscopy areas}

For smooth conduction of the interventional pulmonology procedures, a reorganization of the bronchoscopy areas was done. A separate designated donning area prior to entry in the bronchoscopy suite was made with one nursing staff stationed there to assist in donning. Similarly, a designated doffing area at the other end of the bronchoscopy suite was created along with automatic hand sanitizer dispenser, and biomedical waste collection bins with one nursing staff. Personal protective equipment, including N95 masks, were arranged for all the staff. All healthcare personnel involved in the procedures were given training regarding proper donning and doffing of the personal protective equipment. As the re-use of masks was being practised in certain other areas of the hospital, this practice was completely stopped in the bronchoscopy suite. If more than one procedure was planned on any day, a disposable gown and triple-layered surgical mask were additionally used during the first procedure and discarded after use. The complete plan of the procedure, including the role of each member, was discussed within the team, outside the bronchoscopy suite prior to the procedure, to minimize the procedure duration. Sodium hypochlorite solution (1\%) was used for cleaning all surfaces before and after each procedure.

\section{Technical performance of the procedure}

Patients were shifted to bronchoscopy room with a triple-layer surgical mask in place. Video Bronchoscopy and EBUS were performed under conscious sedation and local anaesthesia by intratracheal instillation of $2 \%$ lignocaine technique. For semirigid thoracoscopy, conscious sedation along with $1 \%$ lignocaine infiltration was used. For upper airway anaesthesia, 2\% nasal lignocaine jelly and $10 \%$ lignocaine spray into oropharynx were used. The patient was covered with a polypropylene sheet during the procedure using side rails of the trolley. The bronchoscope was inserted through a linear slit made in the polypropylene sheet allowing only the bronchoscope to pass while avoiding any droplets to come out (Figure 1). Low flow oxygen (up to $2 \mathrm{l} / \mathrm{min}$ ) was used through nasal prongs whenever required. Oxygen inhalation was not routinely used as it may lead aerosolization of nasal secretions. The sheet was discarded with adequate biomedical precautions after the procedure. This polypropylene sheet is being used at our hospital for packing of biohazard patient specimens. This method was initiated as it was considered to be cost-effective as compared to commercially available bronchoscopy or intubation boxes. The interventional pulmonology procedures performed during January 2020 were used as controls for comparison.

\section{Training assessment}

The training of resident doctors in interventional pulmonology procedures was also assessed. The number of procedures performed and observed by residents in Jan 2020 was compared with the number of procedures performed/ observed by residents during four months of lockdown.

\section{Human resources and additional cost assessment}

We also assessed the number of personnel involved and increase in the approximate cost of the procedure pre lockdown and during the lockdown period. Patients underwent RT-PCR for

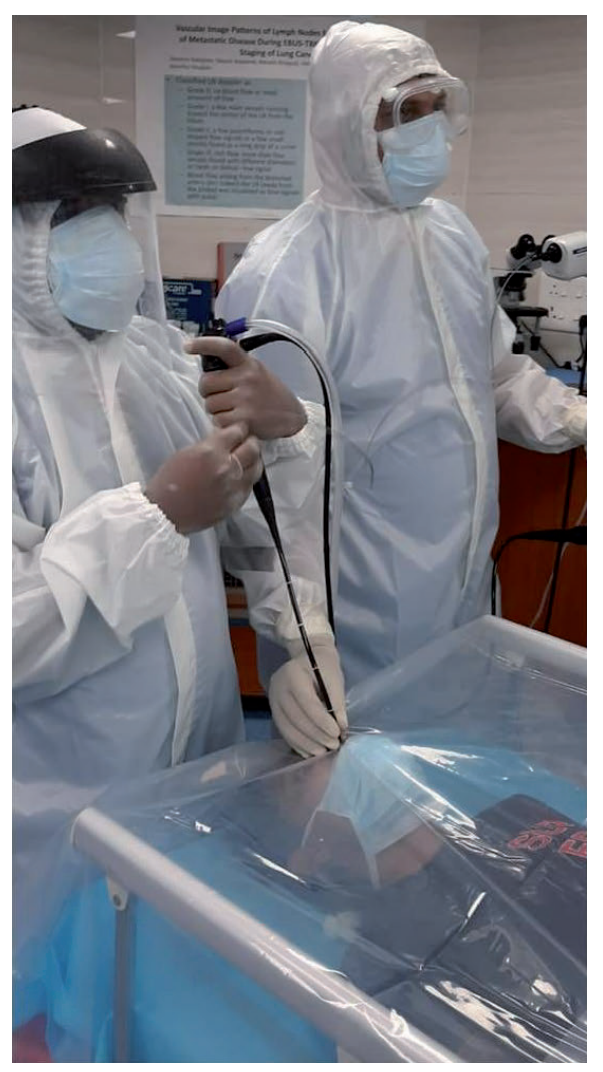

Figure 1. The arrangement made for conducting the bronchoscopy procedures with personal protective equipment including faceshield, using the polypropylene sheet tent. 
COVID 19 prior to the procedure. The health care professionals involved in procedures were monitored closely for symptoms and were advised to undergo testing if they developed any symptoms.

\section{Outcome measures}

The primary outcome was the number of procedures performed during the lockdown as compared to pre-lockdown status. Secondary outcome measures included diagnostic yield, number of procedures performed by residents, the number of personnel involved and additional cost of the procedure.

\section{Statistical analysis}

The data was recorded in a predesigned proforma and entered into the excel sheet for analysis. Statistical analyses were performed using the STATA statistical analysis software (StataCorp. 2017. Stata Statistical Software: Release 15. College Station, TX, USA) Continuous variables were expressed as mean and standard deviation (if normally distributed) while categorical variables were expressed as percentages.

\section{Results}

Twenty-nine procedures were performed during the four months period of the lockdown compared to 174 procedures during one month before lockdown. The mean age of the 29 subjects was 49.1 years (range $19-83$ years), and 24 subjects (82.7\%) were male. Twenty-two flexible bronchoscopies $(75.8 \%)$, four semirigid thoracoscopies (13.7\%) and three EBUS-TBNA (10.3\%) were carried out during this period. Details of the procedures are given in Table 1. Twenty-two patients underwent diagnostic testing for COVID-19 prior to procedures. The reason for not performing COVID-19 testing for seven procedures was that these procedures were performed in the initial one week of lockdown, and there were no clear institutional guidelines for the same at that time. Among these, 23 of the procedures were for the diagnostic indication (79\%), and six were therapeutic (20.6\%). The procedures carried out during lockdown were mainly for suspected infections (41.3\%) and neoplasms (31\%).
The diagnostic yield in suspected neoplasm was $100 \%$ while for suspected infections was $58.3 \%$. Additionally, nine admitted patients, referred for bronchoscopy, underwent an image-guided biopsy which was diagnostic in all cases.

The results during lockdown were compared to the procedures carried out during Jan 2020 (pre lockdown) when 174 procedures were carried out in one month including 119 bronchoscopies (68.3\%), five semirigid thoracoscopy (2.8\%) and 50 EBUS $(28.7 \%)$ were performed. Among these, 155 (89\%) were for diagnostic indication, and $19(11 \%)$ were therapeutic. The clinical indication was suspected neoplasm in $45(25.8 \%)$, and suspected infections in 84 (48.2\%). The diagnostic yield was $53.3 \%$ for suspected neoplasm and $51.1 \%$ for suspected infections. The comparison of diagnostic yield pre and during the lockdown period is shown in Figure 2.

For the 29 procedures carried out over four months of lockdown, none were carried out independently by residents. Six resi-

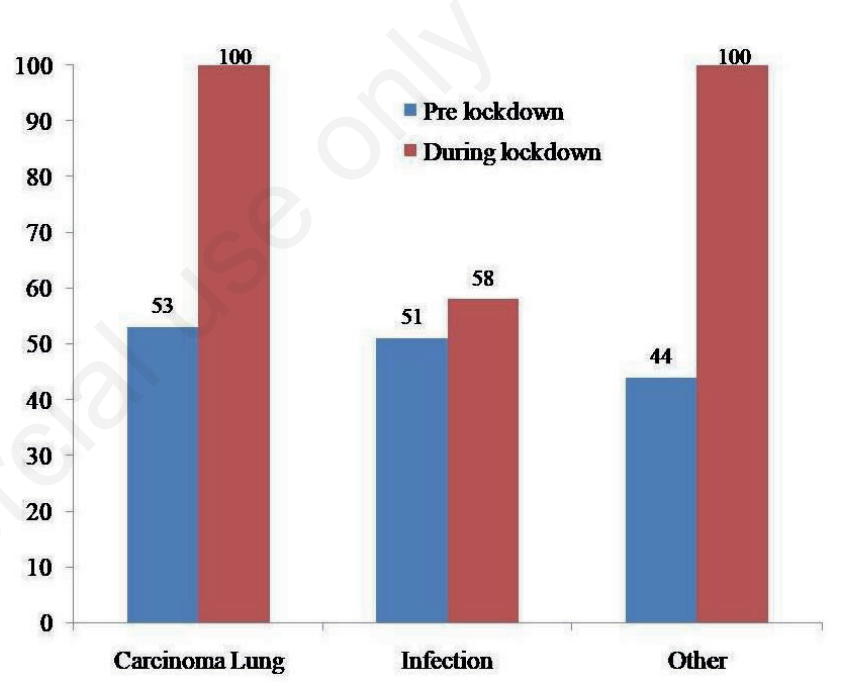

Figure 2. Comparison of yield of the procedures for various indications.

Table 1. Number, indication and yield of the interventional pulmonology procedures.

\begin{tabular}{lcc} 
Total procedures & $\begin{array}{c}\text { Jan } 2020 \\
\text { (pre lockdown) }\end{array}$ & $\begin{array}{c}24 \text { Mar } 2020-23 \text { Jul } 2020 \\
\text { (during lockdown) }\end{array}$ \\
Clinico-radiological diagnosis & 174 & 29 \\
Suspected neoplasm & & $9(31 \%)$ \\
Suspected infections & $45(25.8 \%)$ & $12(41.3 \%)$ \\
Others & $84(48.2 \%)$ & $8(27.5 \%)$ \\
\hline Procedural indication & $45(25.8 \%)$ & $23(79 \%)$ \\
Diagnostic & $155(89 \%)$ & $6(20.6 \%)$ \\
Therapeutic & $19(11 \%)$ & $22(75.8 \%)$ \\
Procedures performed & $119(68.3 \%)$ & $3(10.3 \%)$ \\
Flexible bronchoscopy & $50(28.7 \%)$ & $4(13.7 \%)$ \\
EBUS & $5(2.8 \%)$ & $9 / 9(100 \%)$ \\
Semirigid thoracoscopy & $24 / 45(53.3 \%)$ & $7 / 12(58.3 \%)$ \\
Diagnostic yield & $43 / 84(51.1 \%)$ & $3 / 3(100 \%)$ \\
Bronchogenic carcinoma & $20 / 45(44.4 \%)$ & \\
Infections & & \\
Others &
\end{tabular}


dents assisted these 29 procedures while during one month in January 2020, four residents were involved in the procedures as a part of their training. Seventy-nine of these procedures $(45.4 \%)$ were carried out by residents independently while consultant incharge along with the residents carried out 95 (54.5\%).

Prior to the pandemic, the number of personnel required for around eight procedures per day was six (two doctors, two nurses, one operation theatre technician, one hospital attendant) which markedly increased during the pandemic. The number of personnel involved during the procedure was eight during the pandemic, including two doctors, two assisting nurses, one operation theatre technician, one donning nurse, one doffing nurse and one hospital attendant. As only one-two procedures were carried out in a day, the workforce required per procedure increased from 0.75 to $4-8$.

Additional cost per procedure also increased due to the need for PPE as well as COVID-19 testing. The cost of all PPE per individual was 20 USD, and COVID-19 testing was 35 USD, thus considering two cases per day, the additional cost came out to be 135 USD per procedure.

\section{Discussion}

There has been a significant change in medical practices to adapt to the challenges posed by COVID 19. We reoriented our interventional pulmonology services according to the recommendations [8]. The number of procedures performed markedly reduced during the lockdown. Our approach also shifted to more image-guided procedures for the patients where we could have performed a radial EBUS guided biopsy in pre-COVID times. All procedures were carried out wearing full PPE including N95 respirator, coverall protective covering, face shield, double gloves and long shoe cover.

There has been a change in practices among various medical specialities to adapt to the challenges posed by COVID 19. In a review of the impact of COVID 19 on the management of cancer patients, the changes in the practices included avoiding hospitalization when not strictly necessary, short outpatient department stays, development of teleconsultation services, surface disinfection, use of personal protective equipment (PPE) and testing for COVID-19 in newly diagnosed cancer patients or patients in need of chemotherapy, even if asymptomatic [10]. Similarly, for urology services, the measures advised by Research Urology Network include management of emergent urological conditions only, postponement of prostate biopsy, flexible cystoscopy, replacement of ureteral stents and intravesical therapy for low or intermediate risk bladder cancer, pre-admission telephone triage, strict use of PPE and nasopharyngeal swab for all patients [11].

The procedures were mostly carried out for patients with urgent indications of bronchoscopy such as suspected lung cancer, mediastinal or hilar adenopathy or suspected infection in immunocompromised patients. Other modalities of diagnosis, which generate lesser aerosols like diagnostic pleural aspiration, an imageguided biopsy of the suspected lesion, fine needle aspiration cytology (FNAC) of the peripheral lymph node were preferred over interventional procedures wherever feasible. The significant reduction in the number of procedures being carried out can be attributed to the limited access of patients due to lockdown, along with an emphasis on non-aerosol generating procedures for diagnosis.

The yield of interventions during lockdown showed an increase from pre lockdown period. The improvement in diagnostic yield can be attributed to better patient selection in view of thor- ough assessment prior to intervention, to avoid procedures where alternative methods were likely to yield better results.

Reorganization of the entire interventional pulmonology unit was undertaken to ensure the protection for the bronchoscopy team. Creation of donning and doffing areas was done, and multiple lectures with visual aids were carried out for the entire team to train them in correct infection control practices. Various options, including the aerosol box, were considered to reduce the risk of infection during the procedure. As these methods were expensive, an indigenous method was developed as described in the Methods section above. No member of the team contracted COVID-19 during these four months.

For procedure during COVID times, an increase in the workforce was required in the form of one nurse each for donning and doffing areas. The cost of PPE for eight people who were part of the team increased the cost per procedure. An average of eight procedures was carried out on each bronchoscopy day pre lockdown, but this reduced to one-two during the lockdown. All of these factors contribute to an increase in the cost per procedure. Hence, all factors such as expected diagnostic yield, the urgency of the procedure and possibility of combining urgent and routine procedures should be taken into account to reduce cost. The RT-PCR prior to the procedure, use of polypropylene sheet during the procedure, increase in the number of staff required, reduction in the number of procedures per day and cost of PPE for the entire team contributed to the increased cost per procedure.

COVID-19 has affected residency programs across specialities. In a 25 -item online survey done among 351 urology residents in Italy, it was found that involvement in on-call duties had reduced among $41.1 \%$ of residents. This proportion was $44.2 \%$ for minimally invasive surgery, $74.1 \%$ for diagnostic interventions, $81.2 \%$ for outpatient visits, $62.1 \%$ for endoscopic procedures and $57.8 \%$ for open surgery [12]. Various authors have raised similar concerns among other residency programs like orthopaedics and otorhinolaryngology $[13,14]$. In pulmonary medicine practice also, the impact is obvious. Our institute has a robust residency program for the last nine years. To continue training, we had to make changes like the institution of online seminars, journal clubs and case discussions. The demand for COVID care areas has resulted in a loss of departmental training for $25 \%$ of residents in our department at any given time as a quarter of all residents are exclusively posted in COVID areas. Training for flexible bronchoscopy and other interventional procedures constitute the backbone of any interventional pulmonology department; however, a drastic reduction in the number of cases has resulted in a loss of essential training for residents. In four months, only six residents were able to attend the procedures, of which half would have been carried out by them under the supervision and rest observed in the pre-COVID era. This is likely to cause a significant impact and a possible long term solution to avoid such a situation is the initiation of simulator-based training; however, there is no substitute for realcase based learning.

\section{Conclusions}

COVID 19 has impacted the interventional pulmonology services in various ways ranging from reduction in the number of procedures, an increase in cost per procedure, strict emphasis on infection control practices to a negative impact on training. We need to reorganize the services in light of various guidelines, while also thinking of innovative ideas to reduce cost without compromising the patient safety and infection control practices. 
7. Wong SCY, Kwong RT-S, Wu TC, et al. Risk of nosocomial transmission of coronavirus disease 2019: an experience in a general ward setting in Hong Kong. J Hosp Infect 2020;105 119-27.

1. Hussain A, Yadav S, Hadda V, et al. Covid-19: A Comprehensive review of a formidable foe and the road ahead. Expert Rev Respir Med 2020;14:869-79.

2. Sommerstein R, Fux CA, Vuichard-Gysin D, et al. Risk of SARS-CoV-2 transmission by aerosols, the rational use of masks, and protection of healthcare workers from COVID-19. Antimicrob Resist Infect Control 2020;9:100.

3. COVIDIndia.org [Internet]. COVID-19 tracker updates for India for state wise \& district wise data. Accessed: 12 September 2020. Available from: https://covidindia.org/

4. Madan M, Pahuja S, Mohan A, et al. TB infection and BCG vaccination: are we protected from COVID-19? Public Health 2020;185:91-2.

5. Mohan A, Tiwari P, Bhatnagar S, et al. Clinico-demographic profile \& hospital outcomes of COVID-19 patients admitted at a tertiary care centre in north India. Indian $\mathrm{J}$ Med Res 2020;152:61/9.

6. $\mathrm{Wu} \mathrm{Z,} \mathrm{McGoogan} \mathrm{JM.} \mathrm{Characteristics} \mathrm{of} \mathrm{and} \mathrm{Important} \mathrm{les-}$ sons from the coronavirus disease 2019 (COVID-19) outbreak in China: Summary of a report of 72314 cases from the Chinese Center for Disease Control and Prevention. JAMA 2020; 323:1239-42.

8. Wahidi MM, Shojaee S, Lamb CR, et al. The use of bronchoscopy during the coronavirus disease 2019 pandemic: CHEST/AABIP Guideline and Expert Panel Report. Chest 2020;158:1268-81.

9. Porpiglia F, Enrco Checcucci E, Amparore D, et al. Slowdown of urology residents' learning curve during the COVID-19 emergency. BJU Int 2020;125:e15-7.

10. Raymond E, Thieblemont C, Alran S, et al. Impact of the COVID-19 outbreak on the management of patients with cancer. Targ Oncol 2020; 15: 249-259.

11. Esperto F, Papalia R, Autrán-Gómez AM, et al. COVID-19's impact on Italian urology. Int Braz J Urol 2020;46 26-33.

12. Amparore D, Claps F, Cacciamani GE, et al. Impact of the COVID-19 pandemic on urology residency training in Italy. Minerva Urol Nefrol 2020;72:505-9.

13. Kogan M, Klein SE, Hannon CP, et al. Orthopaedic education during the COVID-19 pandemic. J Am Acad Orthop Surg 2020;28:e456.

14. Quesada PR, Solis SN, Diaz RC, Kraft SM. Otolaryngology residency application during the SARS-CoV-2 (COVID-19) pandemic. Otolaryngol Head Neck Surg 2020;163:89-90. 